\title{
Anti-RAGE antibody ameliorates severe thermal injury in rats through regulating cellular immune function
}

\author{
Xiao-mei ZHU, Yong-ming YAO*, Li-tian ZHANG, Ning DONG, Yan YU, Zhi-yong SHENG \\ Department of Microbiology and Immunology, Burns Institute, First Hospital Affiliated to the Chinese PLA General Hospital, Beijing \\ 100048, China
}

\begin{abstract}
Aim: The receptor of advanced glycation end products (RAGE) participates in a variety of pathophysiological processes and inflammatory responses. The aim of this study was to investigate the therapeutic potential of an anti-RAGE neutralizing antibody for severe thermal injury in rats, and to determine whether the treatment worked via modulating cellular immune function. Methods: Full-thickness scald injury was induced in Wistar rats, which were treated with the anti-RAGE antibody (1 mg/kg, iv) at $6 \mathrm{~h}$ and $24 \mathrm{~h}$ after the injury. The rats were sacrificed on $\mathrm{d} \mathrm{1,} \mathrm{3,} \mathrm{5,} \mathrm{and} \mathrm{7.} \mathrm{Blood} \mathrm{and} \mathrm{spleen} \mathrm{samples} \mathrm{were} \mathrm{harvested} \mathrm{to} \mathrm{monitor} \mathrm{organ}$ function and to analyze dendritic cell (DC) and T cell cytokine profiles. The survival rate was analyzed up to $\mathrm{d} 7 \mathrm{after}$ the injury. Results: Administration of the antibody significantly increased the $7 \mathrm{~d}$ survival rate in thermally injured rats (6.67\% in the model group; 33.33\% in anti-RAGE group). Treatment with the antibody also attenuated the multiple organ dysfunction syndrome (MODS) following the thermal injury, as shown by significant decreases in the organ dysfunction markers, including serum ALT, AST, blood urea nitrogen, creatinine and CK-MB. Moreover, treatment with the antibody significantly promoted DC maturation and T cell activation in the spleens of thermally injured rats.

Conclusion: Blockade of the RAGE axis by the antibody effectively ameliorated MODS and improved the survival rate in thermally injured rats, which may be due to modulation of cellular immune function.
\end{abstract}

Keywords: RAGE; anti-RAGE antibody; burns; thermal injury; multiple organ dysfunction syndrome; cytokine; inflammation; immune response; dendritic cell; $T$ cell

Acta Pharmacologica Sinica (2014) 35: 1167-1176; doi: 10.1038/aps.2014.56; published online 25 Aug 2014

\section{Introduction}

Sepsis, a systemic inflammatory syndrome that can lead to lethal multiple organ dysfunction syndrome (MODS), is a challenging problem in surgical intensive care units. Studies of the pathophysiology of organ failure in sepsis have shown that a dysregulated immune response, or immune paralysis, in which the homeostasis between pro-inflammatory and antiinflammatory reaction is lost plays a key role in the development of MODS ${ }^{[1]}$. The receptor of advanced glycation end products (RAGE), a pattern-recognition receptor that binds a diverse class of ligands - including endogenous molecules or alarmins, such as S100/calgranulins, high mobility group box-1 protein (HMGB1), and amyloid $\beta$-peptide ${ }^{[2-4]}$ - perpetuates the innate immune response and induces the defensive

\footnotetext{
* To whom correspondence should be addressed.

E-mail c_ff@sina.com

Received 2014-01-25 Accepted 2014-05-12
}

immune response $\mathrm{e}^{[5,6]}$. Inhibiting RAGE decreased the levels of multiple pro-inflammatory mediators implicated in the pathophysiology of sepsis and improved short-term survival in rodent models of sepsis ${ }^{[6,7]}$. Clinical trials of interventions designed to modulate the immune response to acute insults and sepsis have been disappointing ${ }^{[8,9]}$; therefore, inhibition of the RAGE axis could provide another strategy for improving clinical outcome. However, it is unclear whether blockade of RAGE can influence the host immune response and survival secondary to severe burns in vivo. In this study, we assessed the effect of an anti-RAGE antibody on MODS and mortality in rats after severe thermal injury. We also assessed the immune functions of dendritic cells (DCs) and T cells from thermally injured rats after treatment with a RAGE-neutralizing antibody.

\section{Materials and methods}

Media and reagents

The anti-murine RAGE neutralizing antibody (anti-RAGE) 
(Beijing Bioss Biotech Co Ltd, China) is a high-affinity rabbitderived polyclonal antibody with a titer greater than 1:20000 (as measured by ELISA). Rabbit albumin was used as a control. Collagenase D from Clostridium histolyticum was purchased from Sigma (St Louis, MO, USA). Ficoll-Paque was purchased from Axis-shield Co, Norway. RPMI-1640, fetal calf serum (FCS), glutamine, penicillin, streptomycin, and HEPES were purchased from TianRunShanda Biotech Co Ltd (Beijing, China). Anti-DC (OX62) MicroBeads (IgG1, clone OX62) were purchased from Miltenyi Biotec $\mathrm{GmbH}$, Germany. Concanavalin A (Con A), thiazolyl blue (MTT), and Triton X-100 were purchased from Sigma. Antibodies used for flow cytometric analysis, including fluorescein isothiocyanate (FITC)conjugated anti-rat major histocompatibility complex (MHC)II (IgG ${ }_{1}$, clone HIS19), phycoerythrin (PE)-conjugated mouse anti-rat CD80 (IgG 1 , $\mathrm{K}$ clone 3H5), PE-conjugated mouse antirat CD86 ( $\operatorname{IgG}_{1}, \mathrm{~K}$ clone $\left.24 \mathrm{~F}\right)$, PE-conjugated mouse anti-rat CD25 (IgG 1 , א clone OX-39), FITC-conjugated mouse anti-rat CD3 (IgM, $\mathrm{k}$ clone 1F4), and mouse anti-rat CD16/CD32 (FC blocker, $\left.F_{c \gamma}-\beta / a-R\right)$ (IgG1b, к clone D34-485) were purchased from BD PharMingen (San Diego, CA, USA). FITC-conjugated anti-rat RAGE (IgG) and FITC-conjugated goat anti-mouse IgG 2A was purchased from Biosynthesis Biotechnology Co, Ltd (Beijing, China). The total RNA isolation system and reverse transcription system were purchased from Promega (Madison, WI, USA). The SYBR Green PCR Master Mix was purchased from Applied Biosystems (Foster City, CA, USA). Enzymelinked immunosorbent assay (ELISA) kits for interleukin (IL)12, tumor necrosis factor (TNF)-a, IL-2, soluble IL-2 receptor (sIL-2R), IL-4, and interferon (IFN)- $\gamma$ were purchased from Biosource (Worcester, MA, USA).

\section{Animal model of thermal injury}

A common technique for producing full-thickness scald injury was used in this study ${ }^{[10]}$. Wistar rats (weight range 250-300 g), purchased from the Laboratory Animal Center, Beijing, China, were housed in separate cages in a temperature-controlled room with $12 \mathrm{~h}$ light and $12 \mathrm{~h}$ darkness and allowed to acclimatize to laboratory conditions for at least $7 \mathrm{~d}$ before undergoing experimental procedures. All animals had free access to water but were fasted overnight prior to the experiment. The rats were anesthetized, and the dorsal and lateral surfaces of the rats were shaved. The rats were then placed on their backs in a protective template exposing $30 \%$ of the total body surface area, and the exposed skin was immersed in $99^{\circ} \mathrm{C}$ water for $12 \mathrm{~s}$. The sham operation rats were subjected to identical treatment but were exposed to room temperature water. The rats were resuscitated $6 \mathrm{~h}$ later with $40 \mathrm{~mL} / \mathrm{kg}$ lactated Ringer's solution (by intraperitoneal injection), followed by an additional $4 \mathrm{~mL}$ at 12, 24, 36, and $48 \mathrm{~h}$ after thermal injury. All experiments were performed according to the National Institute of Health Guide for the Care and Use of Laboratory Animals and were approved by the Scientific Investigation Board of the Chinese PLA General Hospital, Beijing, China.

\section{Experimental design}

Wistar rats were randomly divided into 3 groups: the sham operation group (Sham control), thermal injury group (Burn), and anti-RAGE treatment group (Anti-RAGE). These groups were further divided into four subgroups that were sacrificed on postburn days (PBD) $1,3,5$, and 7 , respectively ( $n=6$ for each group). Anti-RAGE (1 mg/kg) was administered via the dorsal penile vein at 6 and $24 \mathrm{~h}$ after thermal injury in the anti-RAGE treatment group (the dosage and time points were selected based on results from our preliminary experiments). The rats in the sham operation group and the thermal injury group were injected with the same volume of rabbit albumin. Eight untreated rats served as normal controls. The animals were sacrificed on PBD 1, 3, 5, and 7, and blood and spleen samples were harvested aseptically to determine the levels of various organ function markers and cytokines. The spleen samples were divided into two portions: one was used to isolate DC and T cells, and the other was snap-frozen in liquid nitrogen, stored at $-80^{\circ} \mathrm{C}$, and used to isolate total RNA and measure cytokine levels.

To determine the therapeutic efficacy of the RAGEneutralizing antibody for thermally injured rats, 130 Wistar rats were randomly divided into a sham operation group $(n=10)$, a thermal injury group $(n=60)$, and an anti-RAGE antibody treatment group $(n=60)$. The survival rate was recorded up to $7 \mathrm{~d}$ after the procedure.

\section{Measurement of organ function markers}

The blood samples were centrifuged (3000 rounds per minute, $10 \mathrm{~min}$ ) to separate the serum from the blood cells. All of the serum samples were analyzed within $24 \mathrm{~h}$ using a chemistry analyzer (Hitachi 7170, Japan). Liver function was assessed by measuring serum levels of alanine aminotransferase (ALT) and aspartate aminotransferase (AST). Renal function was assessed by measuring serum levels of blood urea nitrogen (BUN) and creatinine ( $\mathrm{Cr}$ ). Myocardial injury was assessed by measuring serum levels of creatine kinase muscle brain isoenzyme (CK-MB).

\section{Isolation of splenic DCs}

The spleen samples were digested with collagenase D and minced, and the mononuclear cells were isolated over a NycoPrep 1.077A density gradient medium, as described previously. Splenic DCs were isolated from mononuclear cells by positive selection using anti-DC (OX62) MicroBeads and a MiniMACS ${ }^{\mathrm{TM}}$ Separator with a positive selection MS column according to the manufacturer's instructions. The DCs were pelleted by centrifugation $(300 \times g, 10 \mathrm{~min})$, and the cell pellet was resuspended in RPMI-1640 (10\% FCS) medium and cultured at $37^{\circ} \mathrm{C}$ in $5 \% \mathrm{CO}_{2}$ in humidified air overnight. The cell culture supernatants were collected to determine cytokine levels.

\section{Isolation of splenic T cells}

Mononuclear cells were isolated as previously described and 
then incubated in nylon wool-packed columns for $2 \mathrm{~h}$ at $37^{\circ} \mathrm{C}$. $\mathrm{T}$ cells were obtained by eluting the columns with $30-40 \mathrm{~mL}$ of RPMI-1640 at a flow rate of one drop per second. In total, $90 \%-95 \%$ of the eluted cells were CD3-positive T cells.

\section{FITC-dextran uptake by DCs}

The DCs were suspended in RPMI-1640, 10\% FCS and 25 mmol/L HEPES, pH 7.4, and incubated with $0.5 \mathrm{mg} / \mathrm{mL}$ of FITC-dextran (Mw=40500; Sigma) for $2 \mathrm{~h}$ at $37^{\circ} \mathrm{C}$ in darkness. The cells were then washed 3 times with ice-cold PBS, $0.1 \%$ BSA and $0.01 \% \mathrm{NaN}_{3}$ and fixed in $10 \%$ formaldehyde in PBS ( $\mathrm{pH}$ 7.2-7.4). The uptake of FITC-dextran by the splenic DCs was assessed by flow cytometry using a FACScan system (BD Biosciences, Mountain View, CA, USA).

\section{Flow cytometric analysis}

DCs $\left(1 \times 10^{5}\right)$ were incubated for $15 \mathrm{~min}$ at $4^{\circ} \mathrm{C}$ in $100 \mu \mathrm{L}$ of $5 \%$ PBS with $0.1 \%$ FCS plus sodium azide (staining buffer) containing PE anti-rat CD80 and PE anti-rat CD86 or FITC anti-rat MHC-II and FITC anti-rat RAGE. FITC-conjugated irrelevant isotype controls were included. T cells $\left(1 \times 10^{5}\right)$ were blocked with $1 \mu \mathrm{g}$ of Fc blocker for $15 \mathrm{~min}$ at $4^{\circ} \mathrm{C}$ in $100 \mu \mathrm{L}$ of $5 \%$ PBS with $0.1 \%$ FCS and then incubated for 20 min in darkness with PE-conjugated CD25 (alpha-chain of IL-2 receptor, IL-2Ra) and FITC-conjugated CD3. The cells were then washed twice with PBS containing 5\% FCS, fixed in PBS containing 10\% formaldehyde ( $\mathrm{pH}$ 7.2-7.4), and examined by flow cytometry using a FACScan system (BD Biosciences, Mountain View, CA, USA).

\section{T cell proliferation assay}

$\mathrm{T}$ cells were suspended to a density of $5 \times 10^{6} / \mathrm{mL}$ in RPMI1640 culture medium supplemented with 10\% FCS, 100 U/mL penicillin, and $100 \mu \mathrm{g} / \mathrm{mL}$ streptomycin and plated in 96-well round-bottomed plates (each sample was plated in 6 replicates). The cells were then treated with $5 \mu \mathrm{g} / \mathrm{mL}$ Con A for $68 \mathrm{~h}$ at $37^{\circ} \mathrm{C}$ in $5 \% \mathrm{CO}_{2}$ in a humidified atmosphere. Next, 10 $\mu \mathrm{L}$ of thiazolyl blue (MTT) $(5 \mathrm{mg} / \mathrm{mL}$ ) was added to each well and the plates were re-incubated for $4 \mathrm{~h}$. Finally, $100 \mu \mathrm{g}$ of acid-isopropanol was added to dissolve the MTT crystals, and the solution was mixed thoroughly by repeated pipetting. The optical density $(O D)$ was measured at $540 \mathrm{~nm}$ using a microplate reader (Spectra MR, Dynex, Chantilly, VA, USA).

\section{SYBR Green Real-time RT-PCR}

Total RNA was extracted from T cells by acid guanidinium thiocyanate-phenol-chloroform extraction according to the manufacturer's instructions. The concentration of the purified total RNA was determined by measuring the absorbance at $260 \mathrm{~nm}$. The IL-2, IL-2Ra, and glyceraldehyde-3-phosphate dehydrogenase (GAPDH) mRNA levels were quantified by SYBR Green 2-step, real-time RT-PCR (all of the samples were quantified in duplicate). After treating with DNase I to remove any potential contaminating DNA, $1 \mu \mathrm{g}$ of total RNA from each sample was reverse transcribed using an oligo-dT primer and Superscript II to generate first-strand cDNA. A PCR mixture was then prepared using the SYBR Green PCR
Master Mix and the primers shown in Table 1. The thermal cycling conditions were: $10 \mathrm{~min}$ at $95^{\circ} \mathrm{C}$ followed by 40 cycles of $95^{\circ} \mathrm{C}$ for $15 \mathrm{~s}$ and $60^{\circ} \mathrm{C}$ for $1 \mathrm{~min}$ on a Sequence Detection System (ABI PRISM 7500, USA). The expression of each gene was normalized to GAPDH mRNA.

Table 1. Sequences of rat primers for SYBR Green PCR.

\begin{tabular}{llll}
\hline \multicolumn{2}{l}{ Target gene } & Oligonucleotide primers $\left(5^{\prime}-3^{\prime}\right)$ & Size \\
\hline IL-2 & Sense & CCATGATGCTCACGTTTAAATTTT & 75 bp \\
& Antisense & TTGCCCAAGCAGGCCACAGAATTG & \\
IL-2R $\alpha$ & Sense & GAATTTATCATTTCGTGGTGGGGCA & \multirow{2}{*}{$98 \mathrm{bp}$} \\
& Antisense & TCTTCTACTCTTCCTCTGTCTCCG & \\
GAPDH & Sense & TGCACCACCAACTGCTTA & $177 \mathrm{bp}$ \\
& Antisense & GGATGCAGGGATGATGTT & \\
\hline
\end{tabular}

\section{Measuring cytokine levels ELISA}

Levels of HMGB1, IL-12, TNF-a, IL-2, sIL-2R, IL-4, and IFN- $\gamma$ in serum or cell culture medium were determined by ELISA, according to the manufacturers' instructions. The color reaction was terminated by adding $100 \mu \mathrm{L}$ of ortho-phosphoric acid. Plates were read in a microplate reader (Spectra MR, Dynex, Chantilly, VA, USA). The concentrations of cytokines in the samples were calculated based on standard curves generated using purified recombinant cytokines. All samples were serially diluted twofold in duplicate for analysis.

\section{Statistical analysis}

The primary endpoint in each experiment was survival. Data are expressed as the mean \pm standard deviation (SD). Differences in survival rate were analyzed using a Kaplan-Meier survival plot and the log-rank test. A one-way ANOVA and an unpaired Student's $t$-test were used to determine significant differences between the groups. A $P$ value of 0.05 or less was considered statistically significant.

\section{Results}

Treatment with an anti-RAGE antibody protected rats from severe thermal injury

The survival rate of thermally injured rats was monitored over $7 \mathrm{~d}$. Administering the anti-RAGE antibody at $6 \mathrm{~h}$ and $24 \mathrm{~h}$ after thermal injury provided significant protection, as these rats showed improved survival compared with the thermal injury group (Figure 1). At PBD 7, the survival rate of rats in the thermal injury group was $6.67 \%$, but treatment with the anti-RAGE antibody after thermal injury increased the survival rate to $33.33 \%$ at the same time point $(P<0.01)$.

The level of organ damage after thermal injury was assessed by measuring the levels of serum markers organ function. As shown in Figure 2, thermal injury induced a significant increase in serum levels of ALT and AST over PBD 1-7 (all $P<0.05)$, indicating damage to liver function. Over the same period, we detected elevated serum levels of BUN and $\mathrm{Cr}$, 


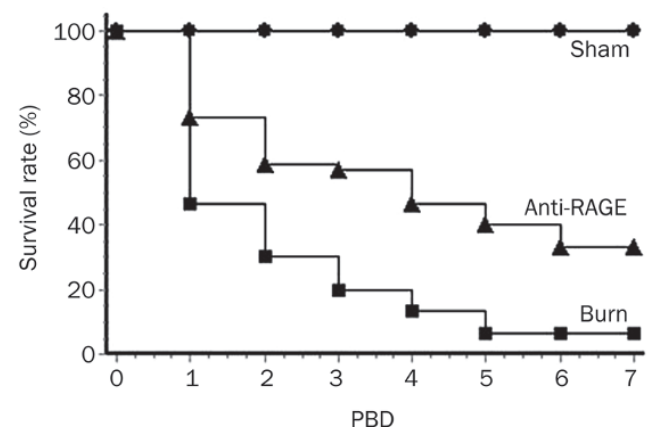

Figure 1. Treatment with the anti-RAGE antibody protected rats from severe thermal injury. The Kaplan-Meier analysis showed a significant difference in the 7 -d survival rate between the anti-RAGE antibody treatment group $(n=60,33.33 \%)$ and the thermal injury group $(n=60$, $6.67 \%, P<0.01)$.

indicating kidney dysfunction, and increased levels of CK-MB in the serum, indicating heart tissue damage (Figure 2) (all $P<0.05)$. However, rats treated with the anti-RAGE antibody exhibited less organ damage than thermally injured animals. Treatment with the anti-RAGE antibody at $6 \mathrm{~h}$ and $24 \mathrm{~h}$ after thermal injury significantly decreased the serum levels of ALT, AST, BUN, Cr, and CK-MB, especially on PBD $3(P<0.01)$ and PBD $5(P<0.05)$, reflecting a reduced level of damage to
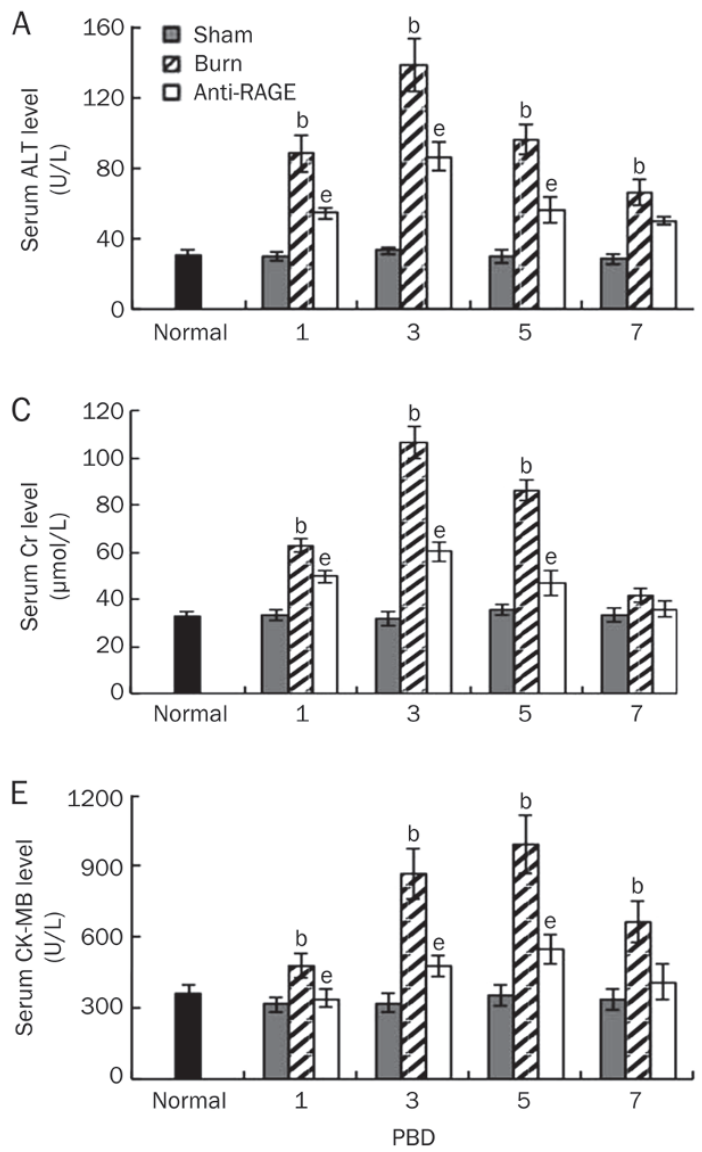

the liver, kidney and heart (Figure 2).

Effect of anti-RAGE treatment on inflammation and immune status in thermally injured rats

We next assessed the levels of inflammatory mediators such as IL-12, HMGB1, and sIL-2R in serum from thermally injured rats. As show in Figure 3, anti-RAGE treatment resulted in a significant increase in IL-12, a cytokine that plays a key role in the development of cell-mediated immunity. In contrast, relatively low levels of IL-12 were detected in serum from thermally injured rats over PBD 1-7 (all $P<0.05)$. The level of sIL-2R (a biomarker reflecting $\mathrm{T}$ cell expansion) in serum from thermally injured rats increased over PBD 3-7. Treatment with the anti-RAGE antibody after thermal injury significantly decreased serum sIL-2R (all $P<0.05$ ). However, the level of HMGB1, an important late inflammatory cytokine that increases after thermal injury, was not markedly affected by administration of the anti-RAGE antibody (all $P>0.20$ ).

The effect of anti-RAGE treatment on RAGE expression on the surface of DCs

DCs, the most important potent antigen-presenting cells, induce and coordinate the host immune response. We therefore determined the expression of RAGE on the surface of DCs isolated from the spleens of thermally injured rats with or without anti-RAGE treatment. As shown in Figure 4, the level
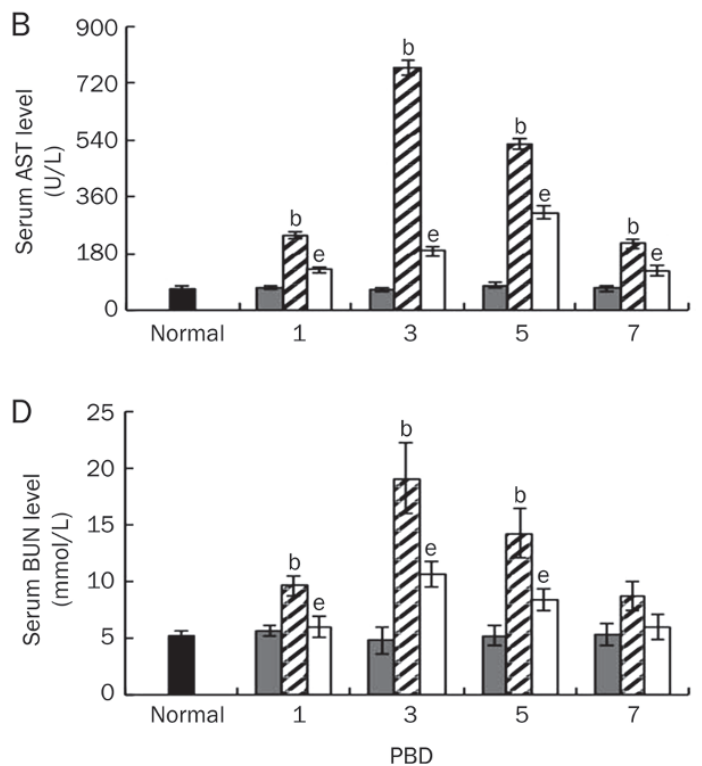

Figure 2. Treatment with the anti-RAGE antibody reduced multiple organ dysfunction caused by thermal injury. Serum was separated from blood samples from rats in the normal control, sham control, thermal injury, and anti-RAGE treatment groups by centrifugation (3000 rounds per minute, $10 \mathrm{~min}$ ). The levels of ALT, AST, BUN, Cr, and CK-MB in each serum sample were determined using a chemical analyzer. The results are shown as the mean \pm SD $(n=6)$. ${ }^{b} P<0.05$ vs the sham control group. ${ }^{e} P<0.05$ vs the thermal injury group. 

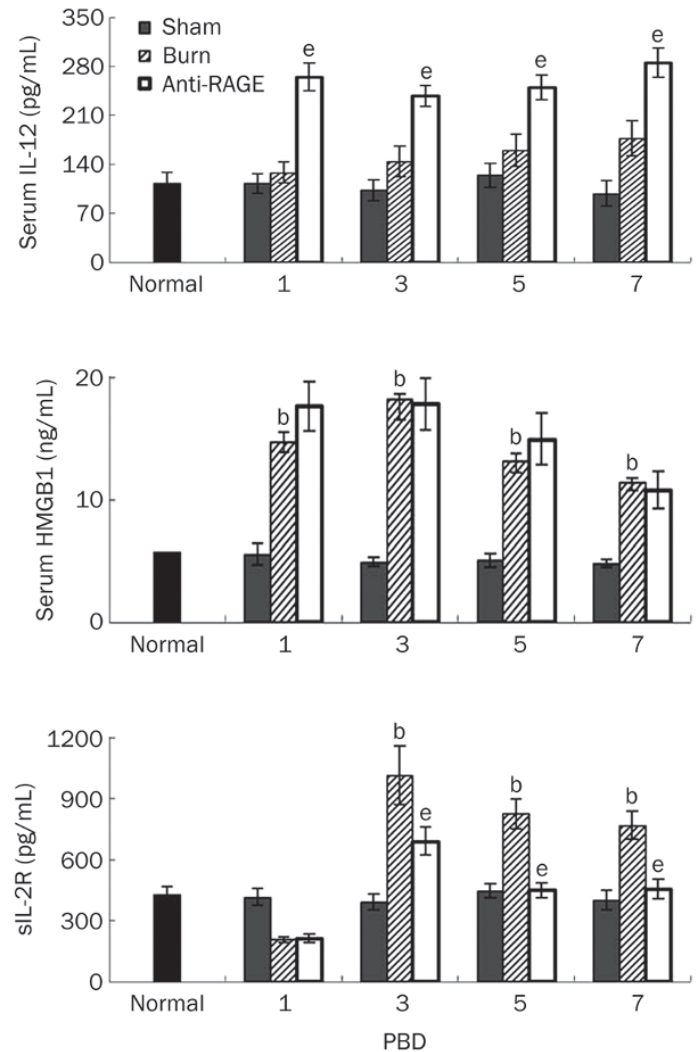

Figure 3. Effect of anti-RAGE antibody treatment on inflammation and the immune status of rats after thermal injury. The serum levels of HMGB1, IL-12, and SIL-2R on PBD 1, 3, 5, and 7 were determined by ELISA. The results are shown as the mean $\pm \operatorname{SD}(n=6)$. ${ }^{\mathrm{b}} P<0.05$ vs the sham control group. ${ }^{\mathrm{e}} \mathrm{P}<0.05$ vs the thermal injury group.

of RAGE expression on the surface of the DCs was significantly higher in thermally injured rats compared to the normal or sham rats. Treatment with the anti-RAGE antibody after thermal injury resulted in a down-regulation of RAGE (Figure 4).

Phenotypic and functional changes in DCs in thermally injured rats following anti-RAGE treatment

We next evaluated the function of DCs from thermally injured rats with or without anti-RAGE treatment. We analyzed the expression of costimulatory molecules including CD80, CD86, and MHC-II, on the surface of DCs and the presence of cytokines, including IL-12 and TNF- $a$, in cell culture supernatants, as well as the capacity for antigen uptake to assess DC maturation and activation.

As shown in Figure 5, splenic DCs expressed high levels of CD86 over PBD 1-7 compared to DCs from sham control rats. However, these DCs expressed only slightly higher levels of MHC class II on PBD 1 and expressed similar levels of CD80 compared to the control, indicating that the splenic DCs induced by thermal injury matured abnormally. Blockade of RAGE significantly enhanced the expression of CD80 and MHC class II on DCs, particularly on PBD 1-3 $(P<0.01)$. CD86
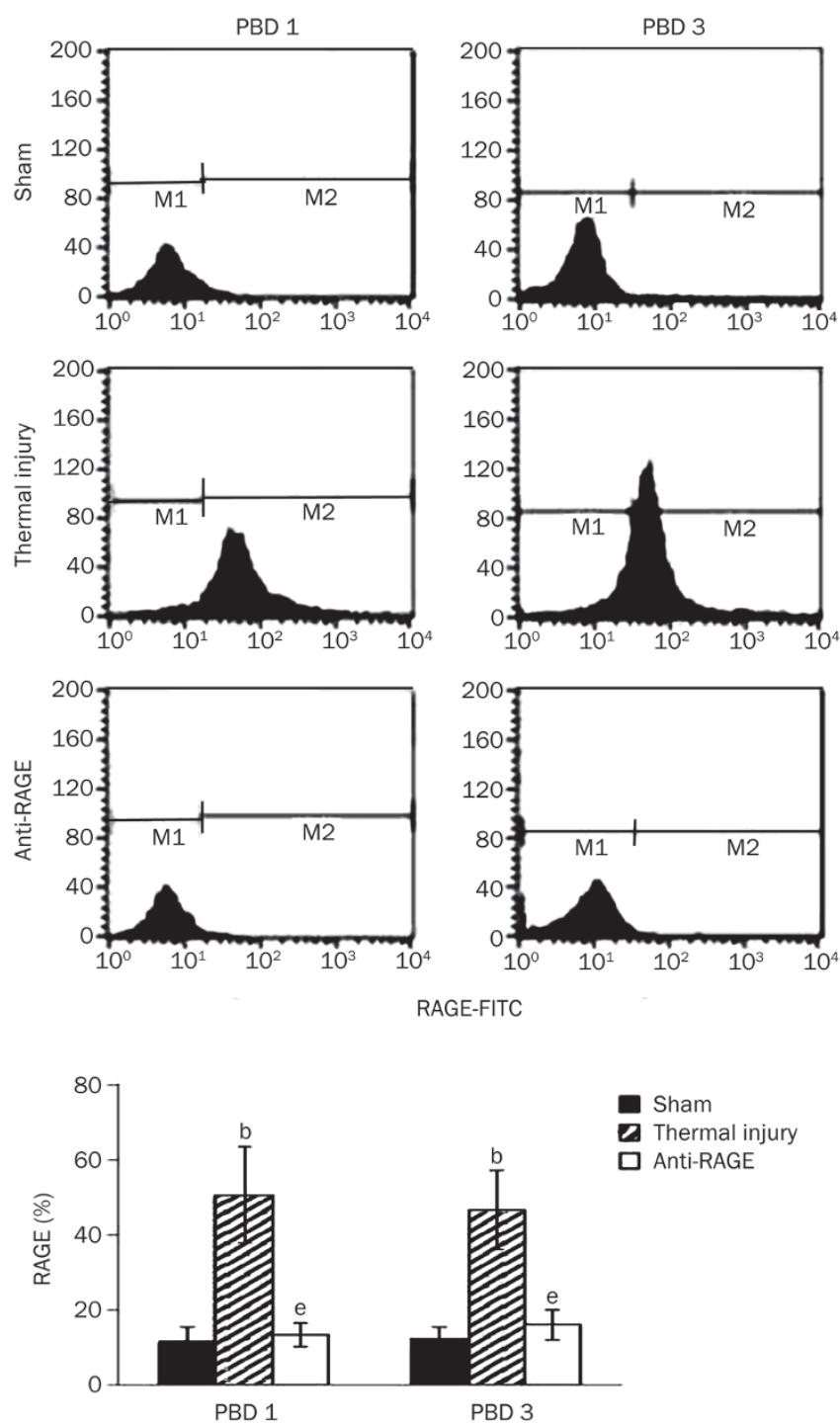

Figure 4. The effect of anti-RAGE treatment on the level of RAGE expression on the surface of DCs. The level of RAGE expression on the surface of DCs isolated from rat spleens on PBD 1 and 3 was assessed by flow cytometry. The results are shown as the mean \pm SD $(n=6)$. ${ }^{b} P<0.05$ vs the sham control group. ${ }^{e} P<0.05$ vs the thermal injury group.

was expressed at high levels over the entire 7 day period, suggesting that treatment with anti-RAGE could contribute to DC activation following severe thermal injury.

We also assessed DC functionality by evaluating uptake of FITC-dextran (Figure 6). While DCs isolated from the normal control and sham rats exhibited normal levels of FITC dextran uptake, the ability of the DCs isolated from thermally injured to take up FITC dextran decreased gradually, suggesting that these DCs had undergone maturation. Treatment with the anti-RAGE antibody further reduced the ability of the DCs isolated from thermally injured rats to take up antigen, especially on PBD $1(P<0.05)$.

Next, we assessed the ability of DCs to produce IL-12 and TNF- $\alpha$, which are markers of mature DC function and key 

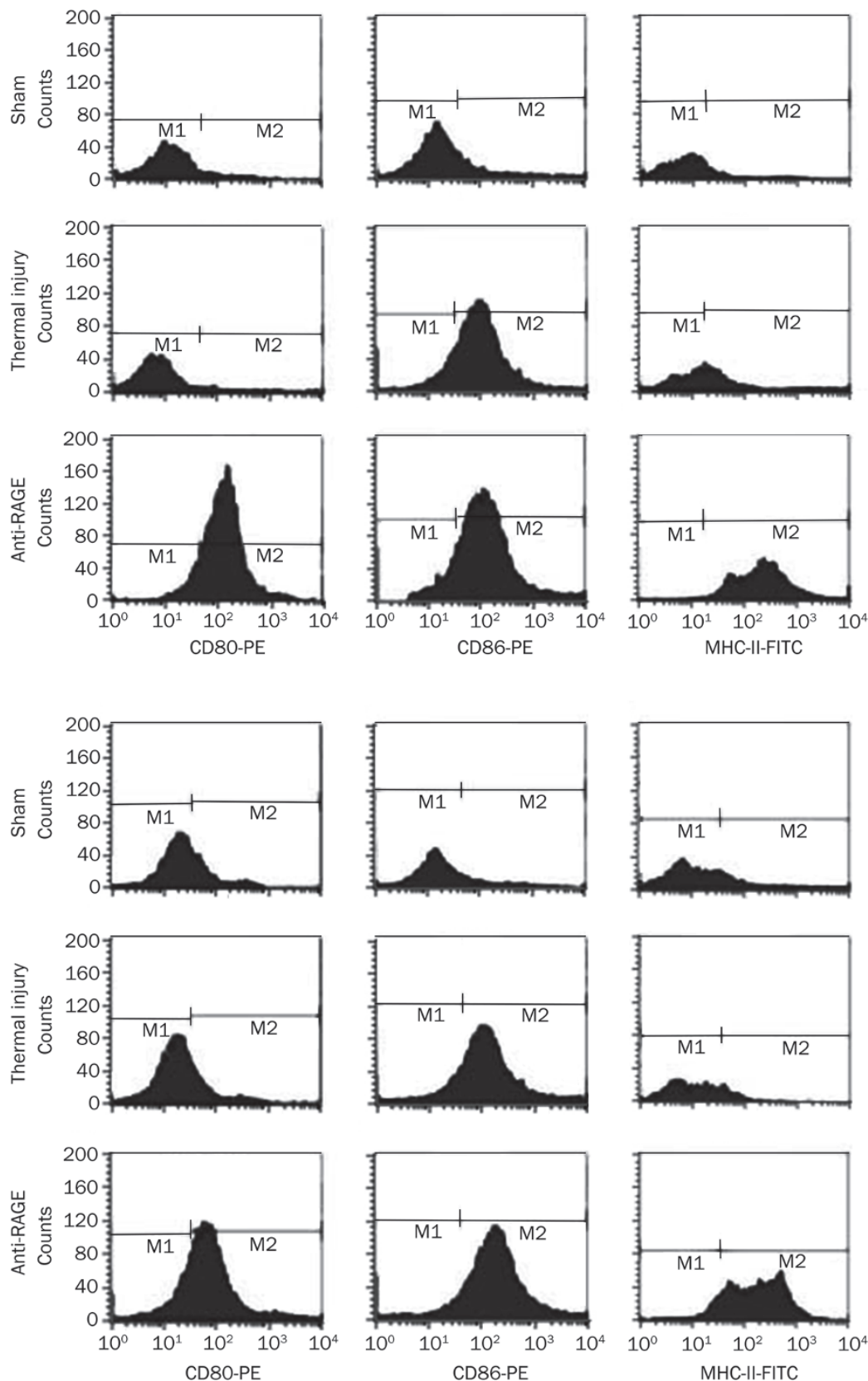
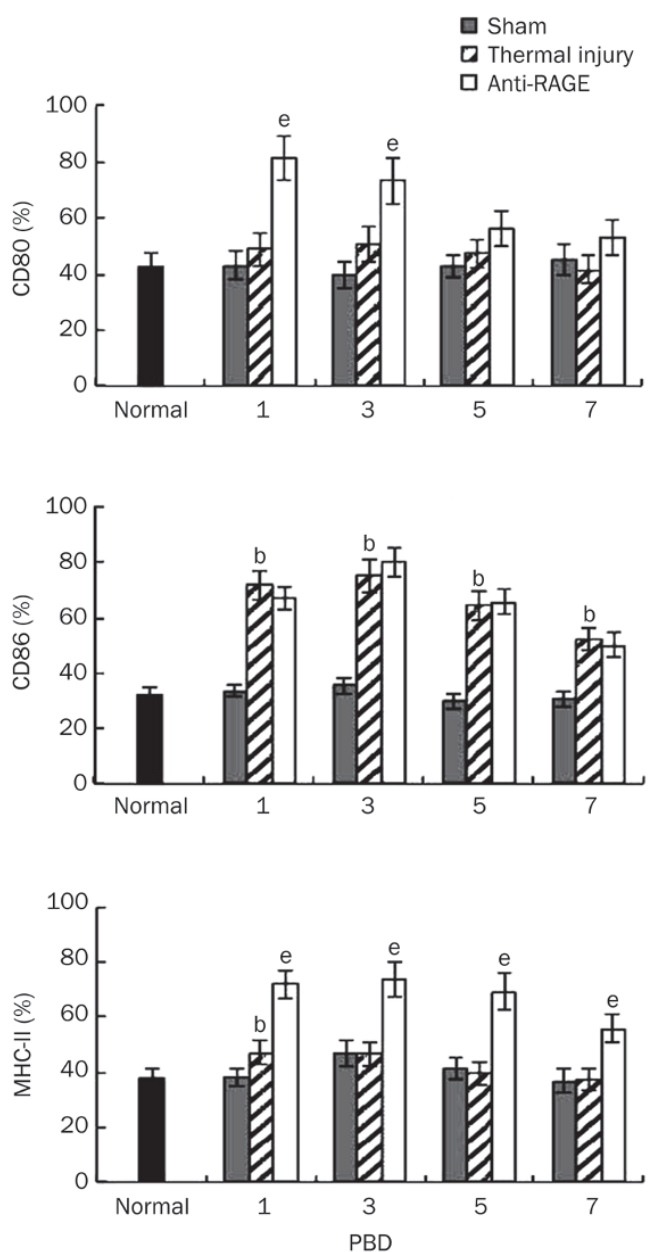

Figure 5. Treatment with anti-RAGE antibody enhanced phenotypic maturation of DCs after burn injury. The phenotypic maturation of DCs was assessed by flow cytometry. DCs were isolated from the rat spleens on PBD 1, 3, 5, and 7 and stained with PE anti-rat CD80 and PE anti-rat CD86 or with FITC anti-rat MHC-II. Representative data plots are shown. The results are shown as the mean \pm SD $(n=6)$. ${ }^{\mathrm{b}} P<0.05 \mathrm{vs}$ the sham control group. ${ }^{\mathrm{e}} P<0.05$ vs the thermal injury group.

mediators for Th1 cell polarization. As shown in Figure 7, compared to the sham control group, DCs isolated from rats in the burn injury group secreted slightly higher levels of IL-12 secretion and decreased levels of TNF-a over PBD 1-7. DCs isolated from rats that were treated with the anti-RAGE antibody after thermal injury secreted significantly increased levels of IL-12 and TNF-a over PBD 1-7 (all $P<0.01$ ), indicating that DC maturation and function in thermally injured rats was improved by treatment with the RAGE-neutralizing antibody.

Effect of anti-RAGE treatment on $\mathrm{T}$ cell activation after burn injury

To understand the potential effect of anti-RAGE antibody treatment on $\mathrm{T}$ cell-mediated immunity, we analyzed $\mathrm{T}$ cell proliferation and cytokine production. Proliferation in response to Con A was significantly decreased in T cells iso- 


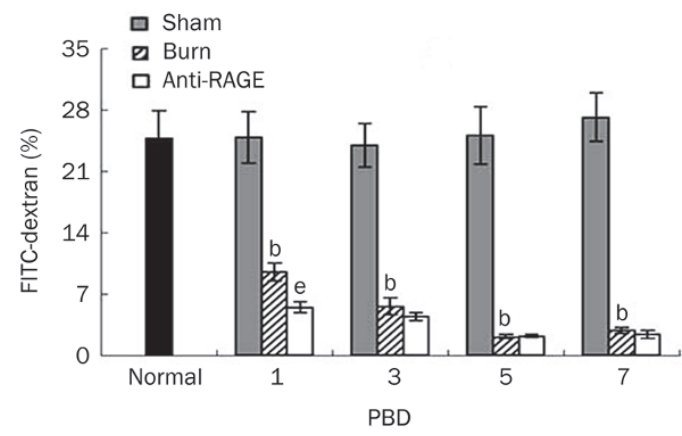

Figure 6. The effect of RAGE-neutralizing antibody treatment on antigen uptake by DCs isolated from thermally injured rats. Splenic DCs isolated from the rat spleens on PBD 1, 3, 5, and 7 were incubated with FITCdextran $(0.5 \mathrm{mg} / \mathrm{mL})$ for $2 \mathrm{~h}$ at $37^{\circ} \mathrm{C}$ in darkness, and the uptake of FITCdextran was assessed by flow cytometry. The results are shown as the mean \pm SD $(n=6) .{ }^{\mathrm{b}} P<0.05$ vs the sham control group. ${ }^{\mathrm{e}} P<0.05$ vs the thermal injury group.
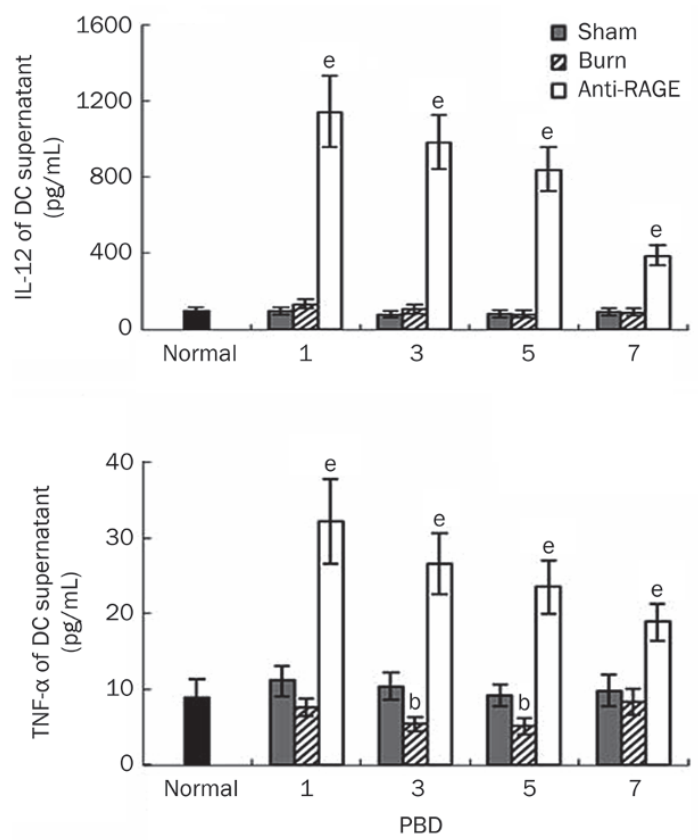

Figure 7. Cytokine secretion by DCs isolated from rats treated with the anti-RAGE antibody after thermal injury. Splenic DCs isolated from rats on PBD 1, 3, 5, and 7 were cultured in RPMI-1640 medium containing 10\% FCS at $37{ }^{\circ} \mathrm{C}$ in $5 \% \mathrm{CO}_{2}$ in a humidified atmosphere overnight. The levels of IL-12 and TNF- $\alpha$ in the DC supernatants were measured by ELISA. The results are shown as the mean \pm SD $(n=6)$. ${ }^{b} P<0.05$ vs the sham control group. ${ }^{e} P<0.05$ vs the thermal injury group.

lated from thermally injured rats over PBD 1-7 compared to T cells isolated from the sham operation group (Figure 8). And as shown in Figure 9, IL-2 mRNA and protein expression, IL-2Ra mRNA expression by T cells, and IL-2Ra expression on the surface of $\mathrm{T}$ cells were somewhat suppressed following thermal injury, and treatment with the anti-RAGE antibody

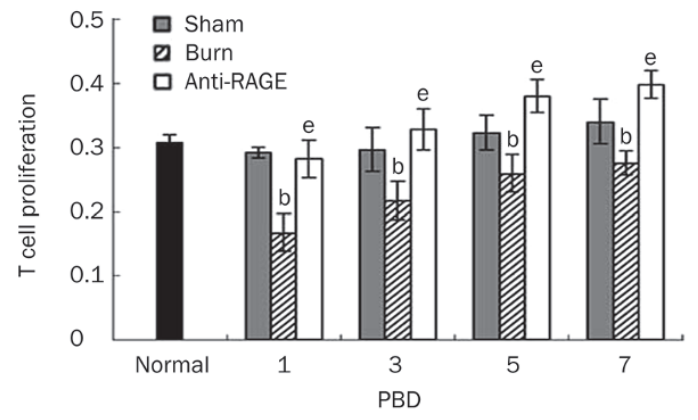

Figure 8. Effect of anti-RAGE treatment on $T$ cell proliferation after thermal injury. T cells isolated from rat spleens on PBD 1, 3, 5, and 7 were cultured with Con $\mathrm{A}$ for $18 \mathrm{~h}$ at $37^{\circ} \mathrm{C}$. T cell proliferation was assessed by an MTT cell proliferation assay. The results are shown as the mean \pm SD $(n=6) .{ }^{b} P<0.05$ vs the sham control group. ${ }^{e} P<0.05$ vs the thermal injury group.

restored normal expression levels (all $P<0.05$ ). Conversely, the level of serum sIL-2R expression decreased initially on PBD 1 and then rapidly increased over PBD 3-7, peaking on PBD 3. Treatment with the anti-RAGE antibody markedly decreased the level of serum sIL-2R levels induced by thermal injury (all $P<0.05)$. These data suggest that blocking RAGE could restore $\mathrm{T}$ cell activation secondary to acute insults.

Effect of anti-RAGE treatment on T cell polarization after thermal injury

Th1 cells produce IFN-y, and Th2 cells produce IL-4. We analyzed the level of these cytokines in T cell supernatants by ELISA to determine the polarization of naive T cells after thermal injury. As shown in Figure 10, following thermal injury, the level of IL-4 produced by T cells in response to Con A increased noticeably over PBD 1-7 (all $P<0.05$ ), whereas the level of IFN- $\gamma$ decreased markedly over the same period (all $P<0.01$ ), indicating that naive $\mathrm{T}$ cells had developed into Th2 cells. Treatment with the anti-RAGE antibody, however, significantly inhibited IL-4 secretion by T cells, particularly on PBD 1 and 3 (all $P<0.05$ ), and increased the release of IFN- $\gamma$ $($ all $P<0.01)$ after thermal injury. These results suggest that blocking RAGE may influence $T$ cell polarization in animals that have undergone thermal injury, inducing the shift to a Th1 phenotype.

\section{Discussion}

Activation of RAGE causes sustained activation of multiple inflammatory pathways that participate in a wide variety of physiological and pathological processes. Recently, RAGE has been recognized as fundamentally important in sepsis due to its role in perpetuating inflammation and its diverse range of ligands ${ }^{[8,11]}$. There is a growing body of evidence from several septic models using genetically modified mice, RAGE neutralizing antibodies, or soluble RAGE suggests the inhibition of RAGE alleviates the inflammatory response and improves survival ${ }^{[7,12-14]}$. In this study, we showed that treatment with an anti-RAGE antibody significantly increased the 

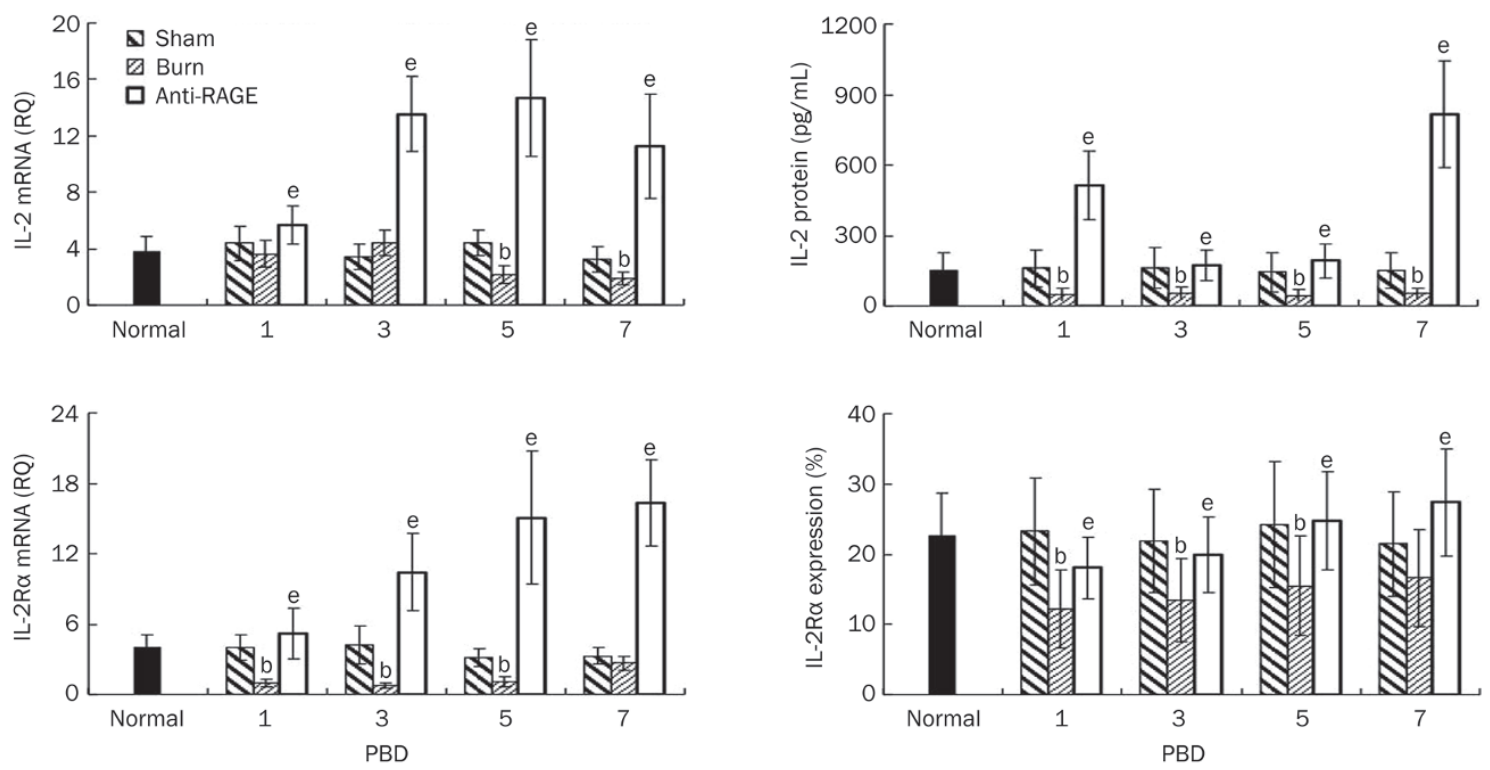

Figure 9. Effect of anti-RAGE treatment on T cell activation after thermal injury. T cells isolated from rat spleens on PBD 1, 3, 5, and 7 were cultured with Con A for $18 \mathrm{~h}$ at $37^{\circ} \mathrm{C}$. The level of IL-2 in the cell culture supernatants was measured by ELISA. IL-2 and IL-2R $\alpha$ mRA levels were measured by real-time PCR. IL-2R $\alpha$ expression on surface of T was also measured by flow cytometry after incubation with PE anti-rat CD25 and FITC anti-rat CD3. The results are shown as the mean \pm SD $(n=6)$. ${ }^{\mathrm{b}} P<0.05$ vs the sham control group. ${ }^{\mathrm{e}} P<0.05$ vs the thermal injury group.
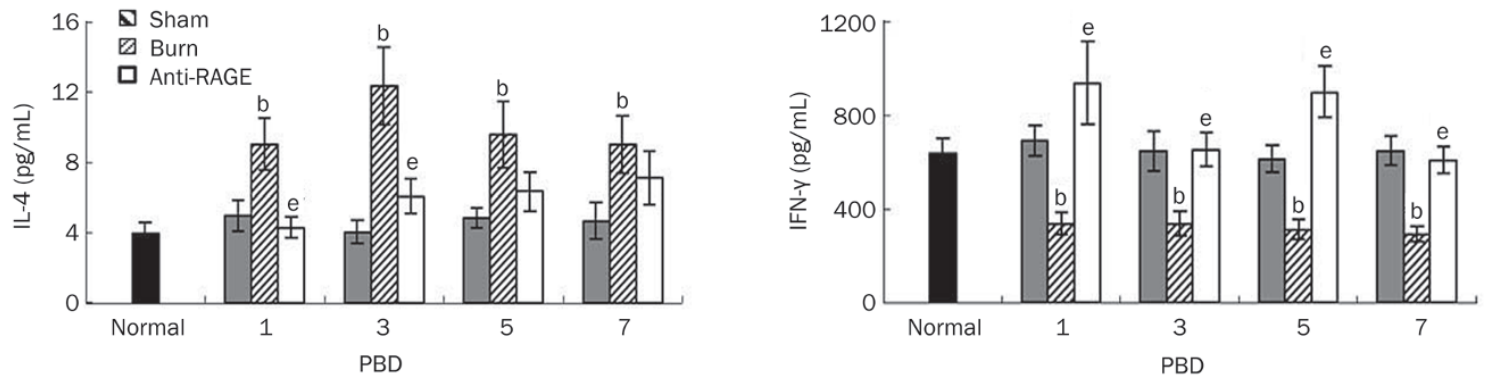

Figure 10. Effect of anti-RAGE treatment on T cell polarization after thermal injury. T cells isolated from rat spleens on PBD 1, 3, 5, and 7 were cultured with Con A for $18 \mathrm{~h}$ at $37^{\circ} \mathrm{C}$. The levels of IFN- $y$ and IL-4 in the culture medium were determined by ELISA to evaluate Th1 and Th2 polarization, respectively. The results are shown as the mean $\pm \operatorname{SD}(n=6) .{ }^{\mathrm{b}} P<0.05$ vs the sham control group. ${ }^{\mathrm{e}} P<0.05$ vs the thermal injury group.

survival rate of rats with severe burns. MODS caused by thermal injury was also dramatically decreased by the blockade of RAGE. These results demonstrate that inhibition of RAGE protected rats from the lethal effects of severe burn-induced sepsis, similar to previous reports. The precise mechanism by which RAGE blockade could reduce the risk of sepsis remains unclear. However, the pathogenesis of sepsis is known to involve countless disturbances of the host immune system. In this study, we focused on the modulation of immune effects by treatment with an anti-RAGE antibody.

Sepsis causes marked immune suppression. We and others have previously shown that dysfunction in DC antigen presentation is a highly significant part of this process ${ }^{[15,16]}$. A previous report demonstrated that RAGE expression can be induced in the presence of its ligands ${ }^{[17]}$, and we show in this study that RAGE is up-regulated on surface of DCs after ther- mal injury. However, treatment with an anti-RAGE antibody resulted in a low level of RAGE expression on the surface of DCs, which correlates well with the effects observed when an anti-RAGE antibody was administered in vivo.

A number of reports have indicated that the RAGE axis plays an important role in DC activation ${ }^{[18]}$. Our data indicate that anti-RAGE treatment had a beneficial effect on DC maturation. We previously showed that severe thermal injury significantly impairs the function of DCs and this effect is related to high levels of HMGB1 in the peripheral blood and spleen ${ }^{[16]}$. HMGB1 is a known RAGE ligand and is involved in sepsis. It is likely that the protective effect of RAGE inhibition in thermally injured rats is due, at least in part, to inhibition of HMGB1. While several in vitro studies have shown that the HMGB1-RAGE interaction modulates DC maturation and activation ${ }^{[19-21]}$, the administration of an anti-RAGE antibody 
in vivo restored DC function after severe burns. These results could be explained by the dual regulatory effect of HMGB1 on DC immune function, which is concentration- and timedependent ${ }^{[22]}$. These results also reflect the intricate regulatory mechanisms operating within host immune cells. Further investigation is needed to elucidate the mechanism by which the RAGE axis modulates the immune response to acute insult followed by sepsis and MODS.

An imbalance in the helper T cell (Th)1/Th2 or cytokine profile has been proposed as a mechanism for septic immunosuppression ${ }^{[23]}$. We previously showed that severe thermal injury decreased $\mathrm{T}$ cell function and induced a shift to the Th1 phenotype $^{[10,16]}$. In this study, we further investigated $\mathrm{T}$ cell proliferation and differentiation in thermally injured rats that were treated with an anti-RAGE antibody. T cells differentiate into effector Th1 or Th2 cells after priming, and the two subsets can be identified by their intracellular cytokine profiles ${ }^{[24]}$. Interestingly, our results show that inhibition of RAGE signaling results in a shift toward the Th1 phenotype compared to rats that suffered thermal injury but received only albumin, as demonstrated by the elevated IFN- $\gamma$ levels and decreased IL-4 levels. Moreover, treatment with a RAGE-neutralizing antibody significantly enhanced $\mathrm{T}$ cell proliferation in response to $\mathrm{T}$ cell mitogen. Treatment with the anti-RAGE antibody significantly restored expression of IL-2 and IL-2Ra. These results indicate that inhibiting RAGE signaling dramatically enhances $\mathrm{T}$ cell proliferation and differentiation. Thus, our findings suggest that RAGE signaling may have a direct effect on $\mathrm{T}$ cell activation or the maturation of DCs that are capable of presenting antigen and stimulating $\mathrm{T}$ cells.

RAGE is a multiligand member of the Ig superfamily of cell surface molecules. RAGE plays a nonredundant role in maturation and immune function of DCs ${ }^{[19]}$. This study shows that the beneficial effects of treatment with an anti-RAGE antibody in thermally injured rats is associated with the restoration of DC function. The precise mechanism of this effect is under investigation.

The finding that treatment with the anti-RAGE antibody resulted in increased levels of IL-12 and HMGB1 was contrary to the outcome we expected. Unfortunately, speculation regarding the reason for this inflammatory state is hampered by the lack of a thorough understanding of cytokine interactions during sepsis. The serum sIL-2R concentration is commonly used as an index for in vivo immune system activation and as a biomarker for identifying patients with severe injury and sepsis who are at risk for developing septic shock ${ }^{[25-27]}$. In our study, treatment with the anti-RAGE antibody decreased the level of sIL-2R, as expected. However, further research is needed to determine the mechanism by which RAGE modulates the host immune response and how anti-RAGE treatment alleviates these effects.

In conclusion, our data show that inhibiting the RAGE axis effectively improves survival in an experimental model of severe thermal injury. The beneficial effects of RAGE blockade on DC maturation and $\mathrm{T}$ cell activation that we observed could open up entirely new avenues for modulating host immune function. Further pre-clinical and clinical investigations will be necessary to evaluate the feasibility of anti-RAGE administration as a therapeutic strategy in humans with lifethreatening sepsis or MODS.

\section{Acknowledgements}

This study was supported by grants from the National Natural Science Foundation of China (№ 81130035, 81272090, and 81372054), the National Basic Research Program of China (No 2012CB518102), the Beijing Nova Program (Z121107002512123), and the State Key Laboratory of Trauma, Burns, and Combined Injury, China (№ SKLKF201103).

\section{Author contribution}

Yong-ming YAO proposed study concepts and acted as guarantor for the integrity of the entire study; Li-tian ZHANG and Xiao-mei ZHU designed and performed the experiments; Ning DONG contributed new reagents and helped with the data analysis; Yan YU helped collect data; Xiao-mei ZHU prepared the manuscript; Zhi-yong SHENG reviewed the manuscript's intellectual content.

\section{References}

1 Ramírez M. Multiple organ dysfunction syndrome. Curr Probl Pediatr Adolesc Health Care 2013; 43: 273-7.

2 Bopp C, Bierhaus A, Hofer S, Bouchon A, Nawroth PP, Martin E, et al. Bench-to-bedside review: The inflammation-perpetuating patternrecognition receptor RAGE as a therapeutic target in sepsis. Crit Care 2008; 12: 201.

3 Creagh-Brown BC, Quinlan GJ, Evans TW, Burke-Gaffney A. The RAGE axis in systemic inflammation, acute lung injury and myocardial dysfunction: an important therapeutic target? Intensive Care Med 2010; 36: 1644-56.

4 Rouhiainen A, Kuja-Panula J, Tumova S, Rauvala H. RAGE-mediated cell signaling. Methods Mol Biol 2013; 963: 239-63.

5 Lin L, Park S, Lakatta EG. RAGE signaling in inflammation and arterial aging. Front Biosci 2009; 14: 1403-13.

6 Luo Y, Li SJ, Yang J, Qiu YZ, Chen FP. HMGB1 induces an inflammatory response in endothelial cells via the RAGE-dependent endoplasmic reticulum stress pathway. Biochem Biophys Res Commun 2013; 438: 732-8.

7 Lutterloh EC, Opal SM, Pittman DD, Keith JC, Tan XY, Clancy BM, et al. Inhibition of the RAGE products increases survival in experimental models of severe sepsis and systemic infection. Crit Care 2007; 11: R122.

8 Webster NR, Galley HF. Immunomodulation in the critically ill. $\mathrm{Br} \mathrm{J}$ Anaesth 2009; 103: 70-81.

9 Rittirsch D, Hoesel LM, Ward PA. The disconnect between animal models of sepsis and human sepsis. J Leukoc Biol 2007; 81: 13743.

10 Zhang LT, Yao YM, Dong N, Dong YQ, Yu Y, Sheng ZY. Relationship between high-mobility group box 1 protein release and T-cell suppression in rats after thermal injury. Shock 2008; 30: 449-55.

11 Bierhaus A, Stern DM, Nawroth PP. RAGE in inflammation: a new therapeutic target? Curr Opin Investig Drugs 2006; 7: 985-91.

12 van Zoelen MA, Schouten M, de Vos AF, Florquin S, Meijers JC, Nawroth PP, et al. The receptor for advanced glycation end products impairs host defense in pneumococcal pneumonia. J Immunol 2009; 182: 4349-56. 
13 Liliensiek B, Weigand MA, Bierhaus A, Nicklas W, Kasper M, Hofer S, et al. Receptor for advanced glycation end products (RAGE) regulates sepsis but not the adaptive immune response. J Clin Invest 2004; 113: 1641-50.

14 Kuhla A, Norden J, Abshagen K, Menger MD, Vollmar B. RAGE blockade and hepatic microcirculation in experimental endotoxaemic liver failure. Br J Surg 2013; 100: 1229-39.

15 Poehlmann H, Schefold JC, Zuckermann-Becker H, Volk HD, Meisel C. Phenotype changes and impaired function of dendritic cell subsets in patients with sepsis: a prospective observational analysis. Crit Care 2009; 13: R119.

16 Zhang LT, Yao YM, Yao FH, Huang LF, Dong N, Yu Y, et al. Association between high-mobility group box-1 protein release and immune function of dendritic cells in thermal injury. J Interferon Cytokine Res 2010; 30: 487-95.

17 Schmidt AM, Yan SD, Yan SF, Stern DM. The biology of the receptor for advanced glycation end products and its ligands. Biochim Biophys Acta 2000; 1498: 99-111.

18 Manfredi AA, Capobianco A, Esposito A, De Cobelli F, Canu T, Monno A, et al. Maturing dendritic cells depend on RAGE for in vivo homing to lymph nodes. J Immunol 2008; 180: 2270-5.

19 Sims GP, Rowe DC, Rietdijk ST, Herbst R, Coyle AJ. HMGB1 and RAGE in inflammation and cancer. Annu Rev Immunol 2010; 28: 367-88.

20 Dumitriu IE, Baruah P, Bianchi ME, Manfredi AA, Rovere-Querini P. Requirement of HMGB1 and RAGE for the maturation of human plasmacytoid dendritic cells. Eur J Immunol 2005; 35: 2184-90.

21 Sterenczak KA, Kleinschmidt S, Wefstaedt P, Eberle N, HewickerTrautwein M, Bullerdiek J, et al. Quantitative PCR and immunohistochemical analyses of HMGB1 and RAGE expression in canine disseminated histiocytic sarcoma (malignant histiocytosis). Anticancer Res 2011; 31: 1541-8.

22 Zhu XM, Yao YM, Liang HP, Xu S, Dong N, Yu Y, et al. The effect of high mobility group box-1 protein on splenic dendritic cell maturation in rats. J Interferon Cytokine Res 2009; 29: 677-86.

23 Lauw FN, ten Hove T, Dekkers PEP, de Jonge E, van Deventer SJH, van der Poll T. Reduced Th1, but not Th2, cytokine production by lymphocytes after in vivo exposure of healthy subjects to endotoxin. Infect Immun 2000; 68: 1014-8.

24 Lanzavecchia A, Sallusto F. The instructive role of dendritic cells on T cell responses: lineages, plasticity and kinetics. Curr Opin Immunol 2001; 13: 291-8.

25 Pierrakos C, Vincent JL. Sepsis biomarkers: a review. Critical Care 2010; 14: R15.

26 Jobin N, Garrel D, Bernier J. Increased serum-soluble interleukin-2 receptor in burn patients: characterization and effects on the immune system. Human Immunology 2000; 61: 233-46.

27 Rubin LA, Nelson DL. The soluble interleukin-2 receptor: biology, function, and clinical application. Ann Intern Med 1990; 113: 61927. 